\title{
Effect of Jianpi Bushen prescription on the expression of SHP-1 and apoptosis-related genes in chemically damaged model mice
}

\author{
D.C. He*, J.J. Xiao*, Y. Zhang, H. Lin, X.J. Ding and Y. Tu \\ Department of Integrated Chinese and Western Medicine, \\ Wuhan General Hospital of Guangzhou Military Region, Wuhan, Hubei, China \\ *These authors contributed equally to this study. \\ Corresponding author: D.C. He \\ E-mail: guilianshang@126.com
}

Genet. Mol. Res. 13 (3): 5957-5963 (2014)

Received January 21, 2014

Accepted March 13, 2014

Published August 7, 2014

DOI http://dx.doi.org/10.4238/2014.August.7.11

\begin{abstract}
We investigated the effect of Jianpi Bushen prescription (JBP) on the expression of the SHP-1 and apoptosis-related genes in chemically damaged model mice and a compound e-jiao slurry (EJS) group (positive control). Kunming mice received an abdominal injection of $100 \mathrm{mg} / \mathrm{kg}$ cyclophosphamide once a day for 3 consecutive days to induce chemical damage. The mice underwent lavage at a suspension of $0.1 \mathrm{~g} / \mathrm{kg}$ low-dose JBP (100\%), high-dose JBP (200\%), and 0.2 $\mathrm{mL} / 10 \mathrm{~g}$ EJS twice a day for 9 days. mRNA and protein expression of SHP-1 in bone marrow mononuclear cells was detected using realtime polymerase chain reaction and Western blot; mRNA expression of B-cell lymphoma 2 (Bcl-2) and Bcl-2-associated X (Bax) protein was detected by in situ hybridization. Expression of SHP-1 and Bax mRNA was significantly upregulated in the model group compared to the control group $(\mathrm{P}<0.05)$. Expression in the low-dose JBP, highdose JBP, and EJS groups was significantly downregulated compared with the model group $(\mathrm{P}<0.05)$. The low-dose JBP group exhibited much lower SHP-1 and Bax mRNA expression levels. Compared with
\end{abstract}


controls, $B c l-2$ mRNA expression was significantly reduced in the model group $(\mathrm{P}<0.05)$. Expression in the low-dose JBP, high-dose JBP, and EJS groups significantly increased compared with the model group $(\mathrm{P}<0.05)$. The low-dose JBP group showed much higher $\mathrm{Bcl}-2$ mRNA expression. Therefore, JBP regulates the expression of the SHP1, $\mathrm{Bax}$, and $\mathrm{Bcl}-2$ genes in chemically damaged mice.

Key words: Animal model; Chemical damage; Bax; Bcl-2 gene; Jianpi Bushen prescription; SHP-1

\section{INTRODUCTION}

Chemical damage significantly endangers human health by suppressing bone marrow and causing cytopenia and immunity hypofunction (Kuhn, 2002; Kemp et al., 2011). Therefore, developing safe, efficient, and low-toxicity drugs that promote hematopoietic function is important. Exploiting the active ingredients in traditional Chinese medicines improves hematopoietic and immune function and significantly prevents or mitigates the harm caused by radiation to human health (Zhang et al., 2012). The Jianpi Bushen prescription [JBP; formerly, Di Gan oral liquid (DGOL)] protects against radiation and chemical damage. Early clinical trials have indicated that JBP can improve peripheral blood and immunologic function. Previous animal experiments established the protection mechanism of JBP against radiation damage to marrow colony stimulating factor, expression of cell cycle genes in the marrow and spleen, and splenic histopathological changes. Changes in apoptosis-related genes (Savli et al., 2011) and uncontrolled signal transduction pathways (Fossett, 2013) may be involved in the complicated mechanisms of chemical damage. Increased expression of B-cell lymphoma 2 (Bcl-2)-associated X (Bax) gene, an apoptosis-accelerating gene, and a decrease in $B c l-2$ mRNA, an anti-apoptosis gene, cause apoptosis in hematopoietic cells (An et al., 2011). The Janus kinase/signal transducer and activator of transcription (JAK/STAT) signaling pathway is a signal transduction pathway involving various hematopoietic growth factors. This pathway significantly affects the growth, proliferation, differentiation, and apoptosis of hematopoietic cells (Kiu and Nicholson, 2012). As a negative regulatory factor in JAK/STAT signaling, the SHP-1 protein inhibits this pathway (López-Ruiz et al., 2011). We examined the effect of JBP on the expression of SHP-1 and apoptosis-related genes to determine the protective mechanism of the Chinese medicinal formula DGOL against radiation damage. DGOL fortifies the spleen and kidneys, reinforces 'qi', and nourishes the blood.

\section{MATERIAL AND METHODS}

\section{Drug preparation}

JBP is composed of prepared Rehmannia root and honey-fried licorice root supplemented with Angelica, Radix astragalus, and tangerine peels. The prescription was prepared by water decoction, filtration, and concentration. JBP was then prepared at concentrations of $100 \%$ ( $1 \mathrm{~g}$ crude drug $/ \mathrm{mL}$ ) and $200 \%$ ( $2 \mathrm{~g}$ crude drug $/ \mathrm{mL}$ ) liquid medicine, sub-packaged, and preserved. Compound e-jiao slurry (EJS) was obtained from Shandong DEEJ (batch number 20101008; Shangdong, China). 


\section{Animal modeling and grouping}

Sixty clean male and female Kunming mice weighing 18-22 g were obtained from the Hubei Province Experimental Animal Research Center.

The mice were adaptively fed for 1 week, and 12 mice were randomly selected as the control group. All other mice received abdominal injections of $100 \mathrm{mg} / \mathrm{kg}$ cyclophosphamide once a day for 3 consecutive days. Reduced appetite and activity were used as the standard for successful modeling of mice. Compared with the control group, the white blood cell count of radiation-damaged model mice was significantly reduced (control, $12.61 \times 10^{9} / \mathrm{L}$; radiation-damaged mice, $7.41 \times 10^{9} / \mathrm{L}$ ). The mice were randomly divided into a model group, a low-dose JBP group ( $100 \%$ concentration), a high-dose JBP group ( $200 \%$ concentration), and a compound EJS group (positive control). The model group underwent lavage with equivalent sodium chloride at a dose of $0.2 \mathrm{~mL} / 10 \mathrm{~g}$ twice a day. The other 3 groups underwent lavage at a $0.1 \mathrm{~g} / \mathrm{kg}$ suspension of lowdose JBP, high-dose JBP, and compound EJS, respectively, at $0.2 \mathrm{~mL} / 10 \mathrm{~g}$ doses twice a day. On day 9 , the mice were sacrificed to collect bilateral femur and tibia bone marrow.

\section{Real-time polymerase chain reaction (PCR)}

Bilateral femur and tibia bone marrow samples were collected and total RNA was extracted using Trizol (Invitrogen, Carlsbad, CA, USA) for the reverse transcription reaction. Primers were designed based on GenBank sequences, and the Primer 5.0 software was used to analyze the primers. Forty PCR cycles were run after 2 min at $94^{\circ} \mathrm{C}$. Each cycle included: $2 \mathrm{~min}$ at $50^{\circ} \mathrm{C}, 10 \mathrm{~min}$ at $95^{\circ} \mathrm{C}$, and $30 \mathrm{~s}$ at $95^{\circ} \mathrm{C}$, followed by holding the reaction at $60^{\circ} \mathrm{C}$. $\beta$-actin was used as a control. Cycle thresholds $(\mathrm{Ct})$ were obtained with the ECO Real-Time PCR System 3.0 (Illumina; San Diego, CA, USA), and differences in genes were calculated using the $2^{-\Delta \Delta \mathrm{Ct}}$ method.

\section{Western blot}

The tissue weight of bone marrow samples was calculated. Radioimmunoprecipitation assay erythrocyte splitting liquor was added to the cell suspension and the mixture was blended. After $5 \mathrm{~min}$ at $4^{\circ} \mathrm{C}$, the supernatant was removed from the centrifuge tube and stored at $-20^{\circ} \mathrm{C}$. Protein concentration was then determined. Electrophoresis loading buffer equivalent to twice the amount of erythrocyte splitting liquor (volume $\mathrm{x}$ protein concentration) was added to the samples. The samples were boiled for $3 \mathrm{~min}$ before electrophoresis (concentrate glue, $20 \mathrm{~mA}$; discrete glue, $35 \mathrm{~mA}$ ) and samples were transferred to a polyvinylidene fluoride membrane. The membrane was immersed in Tris-buffered saline with Tween 20 (TBST; sealing fluid) with $5 \%$ bovine serum albumin, and the mixture was placed on a shaking table at room temperature for $2 \mathrm{~h}$. The sealing fluid was used to dilute primary antibodies, which were incubated with the membrane overnight at $4^{\circ} \mathrm{C}$. Incubated secondary antibodies conjugated to horseradish peroxidase were added, and the membrane was placed on a shaking table at room temperature for $2 \mathrm{~h}$. Enhanced chemiluminescence substrate fluid was added and fluorescence bands were detected by X-ray film squash, development, and fixation. The film was scanned, and the Bandscan software was used to analyze the gray value of glyceraldehyde 3-phosphate dehydrogenase to determine gene expression. 


\section{In situ hybridization}

To samples of nucleated bone marrow cells, $3 \%$ pepsin was added and the cells were diluted with citric acid for digestion at $37^{\circ} \mathrm{C}$ for $30-120 \mathrm{~min}$.

Next, $20 \mu \mathrm{L}$ hybridization solution was added to each sample and the hybridization reaction was conducted at constant temperature $\left(40^{\circ}-42^{\circ} \mathrm{C}\right)$ overnight. A drop of biotinylated mouse anti-dioxin was added, the mixture was incubated at $37^{\circ} \mathrm{C}$ for $60 \mathrm{~min}$ or $20^{\circ} \mathrm{C}$ for $120 \mathrm{~min}$, and 0.5 $\mathrm{M}$ phosphate-buffered saline was used to wash the samples 4 times for 5 min each time. The DBA Color Development Kit (Boster, Wuhan, China) was used; 1 drop each of chromogenic agents A, $\mathrm{B}$, and $\mathrm{C}$ was mixed with $1 \mathrm{~mL}$ distilled water and the mixture was added to the samples. Color was developed for 20-30 min. A Hitachi HV-720 radiography camera (Tokyo, Japan) was used to collect images and the HPIAS-1000 image analysis software was used to collect data.

\section{Statistical analysis}

SPSS 11.0 was used to analyze data. All data are reported as means \pm standard deviation (SD). Differences among groups were examined for statistical significance using the F-test and single-factor analysis of variance between groups as well as the Student $t$-test and least-significant difference analysis of variance between any 2 groups. $\mathrm{P}<0.05$ was considered to be statistically significant.

\section{RESULTS}

\section{SHP-1 expression}

The mice received abdominal injections of cyclophosphamide at a dose of $100 \mathrm{mg} / \mathrm{kg}$ once a day for 3 consecutive days to induce chemical damage (Table 1). The mice underwent lavage with a $0.1 \mathrm{~g} / \mathrm{kg}$ suspension of low-dose JBP, high-dose JBP, and compound EJS for 9 days. Bilateral femur and tibia bone marrow samples were collected, and the mRNA and protein expression of SHP-1 in bone marrow cells was measured by real-time PCR and Western blotting. Compared with the control group, the mRNA and protein expression of SHP-1 was significantly upregulated in the chemically damaged model group $(\mathrm{P}<0.05)$. This expression in the low-dose JBP, high-dose JBP, and compound EJS groups was significantly downregulated compared with that in the chemically damaged model group $(\mathrm{P}<0.05)$. The low-dose JBP group exhibited much lower mRNA and protein expression of SHP-1.

\section{mRNA expression of $B a x$ and $B c l-2$}

The mice received abdominal injections of $100 \mathrm{mg} / \mathrm{kg}$ cyclophosphamide once a day for 3 consecutive days to induce chemical damage (Table 2). The mice underwent lavage with a $0.1 \mathrm{~g} / \mathrm{kg}$ suspension of low-dose JBP, high-dose JBP, and compound EJS for 9 days. Bilateral femur and tibia bone marrow were collected, and the positive cell rate of Bax and Bcl-2 mRNA expression in bone marrow cells was tested by in situ hybridization. Compared with the control group, the positive cell rate of Bax mRNA expression in bone marrow cells significantly increased in the chemically damaged model group $(\mathrm{P}<0.05)$. This rate in the low-dose JBP, 
high-dose JBP, and compound EJS groups was significantly decreased compared with that in the chemically damaged model group $(\mathrm{P}<0.05)$. Low-dose JBP exhibited a much lower rate of Bax mRNA expression in bone marrow cells. Compared with the control group, $B c l-2$ mRNA expression in bone marrow cells was significantly reduced in the chemically damaged model group $(\mathrm{P}<0.05)$. Rates in the low-dose JBP, high-dose JBP, and compound EJS groups were significantly increased compared with the chemically damaged model group $(\mathrm{P}<0.05)$. Lowdose JBP administration resulted in much higher $B c l-2$ mRNA expression in bone marrow cells.

Table 1. Expression of SHP-1 in chemically damaged mice $(\mathrm{N}=12)^{-}$

\begin{tabular}{lccccc}
\hline & Normal group & Low-dose group & High-dose group & Compound e-jiao slurry group & Model group \\
\hline SHP-1 protein & $1.0000 \pm 0.0000$ & $1.0563 \pm 0.0343^{\#}$ & $1.4857 \pm 0.1814^{\#}$ & $1.7269 \pm 0.1773^{\#}$ & $1.9573 \pm 0.2359^{*}$ \\
SHP-1 gene & $1.0390 \pm 0.0244$ & $1.0959 \pm 0.0044^{\#}$ & $1.8143 \pm 0.0024^{\#}$ & $2.4355 \pm 0.0027^{\#}$ & $3.0522 \pm 0.0261^{*}$ \\
\hline
\end{tabular}

Data are reported as means $\pm \mathrm{SD}$. $* \mathrm{P}<0.05$ compared to control group; ${ }^{*} \mathrm{P}<0.05$ compared to chemically damaged model group.

Table 2. Positive cell rates of $B a x$ and $B c l-2$ mRNA expression in murine bone marrow nucleated cells.

\begin{tabular}{lccc}
\hline Groups & $\mathrm{N}$ & Bax & $B c l-2$ \\
\hline Normal & 12 & $37.82 \pm 6.0$ & $57.08 \pm 7.2$ \\
Model & 12 & $46.52 \pm 7.1^{*}$ & $18.21 \pm 6.2^{*}$ \\
High-dose & 12 & $24.86 \pm 5.5^{\#}$ & $30.34 \pm 4.8^{\#}$ \\
Low dose & 12 & $19.78 \pm 2.9^{\#}$ & $52.60 \pm 5.4^{\#}$ \\
Compound e-jiao slurry & 12 & $23.43 \pm 6.3^{\#}$ & $32.18 \pm 7.1^{*}$ \\
\hline
\end{tabular}

Data are reported as means $\pm \mathrm{SD}$ in percentage. $* \mathrm{P}<0.05$ compared to control group; ${ }^{*} \mathrm{P}<0.05$ compared to chemically damaged model group.

\section{DISCUSSION}

JBP is an empirical drug prepared in our laboratory. JBP is composed of a large dose of prepared Rehmannia root and honey-fried licorice root supplemented with Angelica, $R$. astragalus, and tangerine peel. Similar to various other drugs, JBP can fortify the spleen and kidneys and nourish the blood and marrow. Rehmannia glutinosa has the ability to recover the hematopoietic function of bone marrow suppressed by radiation damage as well as significantly promote the proliferation of hemopoietic stem cells and improve immune function (Ma et al., 2010). Angelica polysaccharides significantly promote the proliferation and differentiation of liver hematopoietic cells and myeloid hematopoietic progenitor cells, particularly with reduced peripheral blood cells and suppressed bone marrow. These polysaccharides also improve hematopoietic microenvironments to regulate hematopoiesis (Liu et al., 2010; Hou et al., 2012). $R$. astragalus comprehensively and continuously promotes whole blood cells, restores low red blood cell, white blood cell, and platelet counts in rats, stimulates the secretion of hematopoietic cytokines and hematopoietic system function, and improves bone marrow suppression caused by chemotherapy and radiotherapy (Gao et al., 2006; Zheng et al., 2011). In summary, the spleen and kidneys have the dual role of regulating the proliferation of hematopoietic cells and immune function.

In this study, we examined the effect of JBP on the expression of SHP-1 and apoptosisrelated genes to determine the protective mechanism of the Chinese medicine formula DGOL. SHP-1 is a tyrosine phosphatase that includes the SH2 domain and is a novel candidate as cancer suppressor genes expressed in hematopoietic cells. SHP-1 decreases the phosphorylation 
of downstream signaling proteins, weakens cell growth signals, or activates apoptosis signals to negatively control the differentiation, growth, and proliferation of hematopoietic cells (Nakata et al., 2011). JAK/STAT is one of the most important signal transduction pathways and significantly affects the growth, proliferation, differentiation, and apoptosis of hematopoietic cells (Hankey, 2009). Hematopoietic growth factor mediates intracellular signaling by coupling corresponding receptors via the JAK/STAT pathway to influence DNA expression and the growth and function of hematopoietic cells (Baker et al., 2007). SHP-1 phosphorylation can negatively control JAK activity and thus restrain JAK/STA signaling pathway transmission (Chen et al., 2012). SHP-1 can induce cell apoptosis by regulating and controlling the cell cycle or through the Bax pathway (Hsu et al., 2001). Bcl-2 is one of the most important anti-apoptosis genes, but can also promote apoptosis (Li et al., 2011; Verma et al., 2013). In this study, we determined the mRNA and protein expression levels of SHP-1 and mRNA levels of the apoptosis-related genes Bax and Bcl-2. The mRNA and protein expression of SHP-1 and Bax mRNA increased, while $B c l-2$ mRNA decreased in bone marrow nucleated cells. After JBP treatment, which fortifies the spleen and kidneys, reinforces 'qi', and nourishes the blood, the mRNA and protein expression of SHP-1 and Bax mRNA decreased, while Bcl-2 mRNA increased in bone marrow nucleated cells. Therefore, bone marrow hematopoietic cells were protected from apoptosis, and bone marrow hematopoietic recovery was promoted. Low-dose JBP was more effective than high-dose JBP, indicating that high concentrations of the drug reduced protection against chemical damage and that the large dose of prepared Rehmanniae root and licorice oiliness prevented gastrointestinal absorption. In our future studies, we will examine the specific mechanism of JBP and determine appropriate therapeutic concentrations.

In conclusion, the Chinese medicine compound DGOL protects against myelosuppression and chemical damage. Downregulation of SHP-1 protein expression weakens the inhibition function of the JAK/STAT signaling pathway, enhances expression of anti-apoptosis genes, restrains apoptosis, and facilitates the recovery of bone marrow hematopoiesis.

\section{ACKNOWLEDGMENTS}

Research supported by the Military Medicine Special Task (\#10zyz218) and the National Natural Science Foundation of China (\#81273905).

\section{REFERENCES}

An YS, Lee E, Kang MH, Hong HS, et al. (2011). Substance P stimulates the recovery of bone marrow after the irradiation. J. Cell Physiol. 226: 1204-1213.

Baker SJ, Rane SG and Reddy EP (2007). Hematopoietic cytokine receptor signaling. Oncogene 26: 6724-6737.

Chen KF, Tai WT, Hsu CY, Huang JW, et al. (2012). Blockade of STAT3 activation by sorafenib derivatives through enhancing SHP-1 phosphatase activity. Eur. J. Med. Chem. 55: 220-227.

Fossett N (2013). Signal transduction pathways, intrinsic regulators, and the control of cell fate choice. Biochim. Biophys. Acta 1830: 2375-2384.

Gao QT, Cheung JK, Li J, Chu GK, et al. (2006). A Chinese herbal decoction, Danggui Buxue Tang, prepared from Radix astragali and Radix angelicae sinensis stimulates the immune responses. Planta Med. 72: 1227-1231.

Hankey PA (2009). Regulation of hematopoietic cell development and function by Stat3. Front Biosci. 14: 5273-5290.

Hou H, Bao Y, Li Q and Shi W (2012). Preparation of blood-deficient model and research of Angelica polysaccharide on enriching blood in chickens. Evid. Based. Complement. Alternat. Med. 2012: Article ID 965947.

Hsu HC, Shultz LD, Su X, Shi J, et al. (2001). Mutation of the hematopoietic cell phosphatase (Hcph) gene is associated with resistance to gamma-irradiation-induced apoptosis in Src homology protein tyrosine phosphatase (SHP)-1- 
deficient "motheaten" mutant mice. J. Immunol. 166: 772-780.

Kemp K, Morse R, Sanders K, Hows J, et al. (2011). Alkylating chemotherapeutic agents cyclophosphamide and melphalan cause functional injury to human bone marrow-derived mesenchymal stem cells. Ann. Hematol. 90: 777-789.

Kiu H and Nicholson SE (2012). Biology and significance of the JAK/STAT signalling pathways. Growth Factors 30: $88-106$.

Kuhn JG (2002). Chemotherapy-associated hematopoietic toxicity. Am. J. Health Syst. Pharm. 59: S4-S7.

Li YR, Cao W, Guo J, Miao S, et al. (2011). Comparative investigations on the protective effects of rhodioside, ciwujianoside-B and astragaloside IV on radiation injuries of the hematopoietic system in mice. Phytother. Res. 25: 644-653.

Liu C, Li J, Meng FY, Liang SX, et al. (2010). Polysaccharides from the root of Angelica sinensis promotes hematopoiesis and thrombopoiesis through the PI3K/AKT pathway. BMC Complement. Altern. Med. 10: 79.

López-Ruiz P, Rodriguez-Ubreva J, Cariaga AE, Cortes MA, et al. (2011). SHP-1 in cell-cycle regulation. Anticancer Agents Med. Chem. 11: 89-98.

Ma J, Liang QD, Ma ZC, Wang YG, et al. (2010). Rehmanniae Radix provides most of the free fructose and glucose in Si-Wu-Tang decoction. Drug Discov. Ther. 4: 179-183.

Nakata K, Suzuki Y, Inoue T, Ra C, et al. (2011). Deficiency of SHP1 leads to sustained and increased ERK activation in mast cells, thereby inhibiting IL-3-dependent proliferation and cell death. Mol. Immunol. 48: 472-480.

Savli H, Gluzman DF, Sunnetci D, Zavelevich MP, et al. (2011). Quantitative real time PCR analysis of apoptosis-related gene expression in leukemias in Ukrainian patients. Exp. Oncol. 33: 104-106.

Verma YK, Raghav PK, Raj HG, Tripathi RP, et al. (2013). Enhanced heterodimerization of Bax by Bcl-2 mutants improves irradiated cell survival. Apoptosis 18: 212-225.

Zhang W, Yao H, Wang S, Shi S, et al. (2012). Wnt3a is involved in the early stage of miPSC and mESC haemopoietic differentiation. Cell Biol. Int. 36: 267-271.

Zheng KY, Choi RC, Cheung AW, Guo AJ, et al. (2011). Flavonoids from Radix astragali induce the expression of erythropoietin in cultured cells: a signaling mediated via the accumulation of hypoxia-inducible factor-1 $\alpha$. J. Agric. Food Chem. 59: 1697-1704. 